\title{
Conrad et Simon : une question de poét(h)ique
}

Josiane Paccaud-Huguet

\section{(2) OpenEdition}

Journals

Édition électronique

URL : https://journals.openedition.org/ccs/444

DOI : $10.4000 /$ ccs.444

ISSN : 2558-782X

\section{Éditeur :}

Presses universitaires de Rennes, Association des lecteurs de Claude Simon

\section{Édition imprimée}

Date de publication : 30 juin 2005

Pagination : $37-45$

ISBN : 2914518676

ISSN : $1774-9425$

\section{Référence électronique}

Josiane Paccaud-Huguet, "Conrad et Simon : une question de poét(h)ique », Cahiers Claude Simon [En ligne], 1 | 2005, mis en ligne le 19 septembre 2017, consulté le 01 mars 2022. URL : http:// journals.openedition.org/ccs/444 ; DOI : https://doi.org/10.4000/ccs.444 


\title{
Conrad et Simon : une question de poét(h)ique
}

\author{
Josiane PACCAUD-HUGUET ${ }^{*}$
}

L'une des deux épigraphes du Tramway de Claude Simon fait référence à la célèbre métaphore spectrale du récit dans $A u$ Cour des Ténèbres:

" ...pour lui le sens d'un épisode ne se trouve pas à l'intérieur, comme d'une noix, mais à l'extérieur, et enveloppe le conte qui l'a suscité, comme une lumière suscite une vapeur... " ${ }^{1}$

Cela voudrait-il dire que le sens n'existe pas ? Non, mais plutôt que la signifiance travaille autrement : moins dans la chair du mot qu'en périphérie, par l'effet d'un éclairage latéral sur les particules verbales en suspension dans l'air comme de la vapeur d'eau - c'est du moins ce qu'indique le narrateur conradien :

Les histoires de marins ont une simplicité directe, dont tout le sens tient dans la coque d'une noix ouverte. Mais Marlow n'était pas typique [...] et pour lui le sens d'un épisode n'était pas à l'intérieur comme les cerneaux, mais à l'extérieur, enveloppant seulement le récit qui l'amenait au jour comme un éclat voilé fait ressortir une

\footnotetext{
* Université Lumière-Lyon 2. Josiane Paccaud-Huguet est directrice de la série Conrad de La Revue des Lettres Modernes (Lettres Modernes Minard).

1 J. Conrad, cité par Claude Simon, Le Tramway, Éditions de Minuit, 2001 (ciaprès, Tram.).
} 
brume, à la semblance de l'un de ces halos vaporeux que rend parfois visibles l'illumination spectrale du clair de lune. ${ }^{2}$

Là où Claude Simon parle d'une " architecture purement sensorielle " à propos de son ouvre ${ }^{3}$, Conrad évoque dans le célèbre manifeste qu'est la préface du Nègre du Narcisse la puissance évocatoire du mot écrit : il fallait par exemple que Au cœur des ténèbres continuât de vibrer d'une note sombre à l'oreille du lecteur longtemps après le placage de l'accord final. La deuxième épigraphe du Tramway renvoie à Proust et son idéal d'un effacement du " personnage réel " au profit de l'image ${ }^{4}$. Là encore, il s'agit moins de faire disparaître le personnage de l'écriture romanesque, que de lui infliger une déperdition de substance au profit du spectre lumineux et sonore irradiant de la page écrite qui n'est pas sans rappeler, mutatis mutandis, l'alchimie photographique.

La lecture de Tramway en écho avec Le Jardin des Plantes donne à penser que ce choix est autant une question d'esthétique que d'éthique, une façon de faire place à la dimension de l'hétérogène, susceptible de faire tache dans le sens et touche pour le lecteur. Lucien Dällenbach parle du " pouvoir d'empreinte du texte » inséparable de la " volonté de savoir " du lecteur, le paradoxe étant que cette volonté de savoir se heurte à un impossible à dire qui réussit pourtant à s'écrire : car le texte introduit des questions "impensées parce qu'impensables dans le cadre d'une théorie du texte conçu comme somme de procédés. ${ }^{5}$ ". L'impensé, ce qui n’a pas de nom ni de forme : ce qualificatif tente de cerner la matière composite et pulsatile d'un réel qui produit des éclats dans la langue sans qu'on puisse dire de quoi elle est faite. De ces éclats surgit un silence de mort,

\footnotetext{
2 Joseph Conrad, Au cour des ténèbres, Euvres II, Gallimard, Bibliothèque de la Pléiade, 1985, p. 48 (ci-après, $A C T$ ).

3 Cité par Lucien Dällenbach, "Le tissu de mémoire ", annexe à La Route des Flandres, Éditions de Minuit, coll. "Double », p. 300.

${ }^{4}$ «[...] l'image étant le seul élément essentiel, la simplification qui consisterait à supprimer purement et simplement les personnages réels serait un perfectionnement décisif." "

5 Lucien Dällenbach, op. cit., p. 300.
} 
celui-là même que le cœur des ténèbres conradiennes enserre : l'horreur du fantasme originaire où Kurtz s'abolit ne s'évoque que d'un réseau de chaînes phonématiques associant l'ivoire à l'horreur d'y voir... de trop près.

Tout se passe comme si le principe poétique du texte s'inspirait de la rumeur circulant dans l'air vicié du temps :

Le mot " ivoire " était dans l'air, se murmurait, se soupirait. On aurait dit qu'ils lui adressaient leurs prières. Un relent de rapacité imbécile montait de l'ensemble, comme une exhalaison venue d'un cadavre. (ACT, 75-76)

À la manière des harmoniques soulevées par l'émission d'un son fondamental, le récit se construit en ondes concentriques autour de ce cri au sens indomiciliable. Et quand Marlow, de retour en Europe, va éclairer la Promise de Kurtz sur les derniers mots du disparu, il sent revenir le souffle dangereux qui menace l'édifice des semblants victoriens :

[...] le son de sa voix paraissait avoir pour accompagnement tous les autres sons emplis de mystère, de désolation et de chagrin que j'eusse jamais entendus - le clapotis du fleuve, le frémissement des arbres balancés par le vent, la rumeur des foules, la faible résonance des mots incompréhensibles criés à distance, le chuchotis d'une voix qui parlait d'au-delà du seuil des ténèbres éternelles. $(A C T, 148)$

L'obscurité les répétait en un souffle tout autour de nous, en un souffle qui paraissait s'enfler, menaçant, comme le premier souffle d'un vent qui se lève. "L'horreur ! L'horreur ! " $(A C T, 150)$

L'expérience de $A u$ Cour des ténèbres consiste à refuser à ce mot un sens qui relèverait de l'interprétation, d'un scénario fantasmatique construit autour d'un désir de "ça-voir ". Il n'y a pas grand chose à dire de cette oralité primitive si ce n'est qu'elle jette une lueur étrange sur la présence coloniale en Afrique. L'écriture ne prend son efficacité que du fait qu'elle laisse passer un soupir de savoir venu non pas d'ailleurs, mais du noyau-même de ce que les Victoriens appelaient encore " civilisation " : un savoir qui serait donc extime, pour reprendre le mot forgé par Jacques Lacan. Il me semble que c'est très exactement, et sans doute pour des raisons ana- 
logues liées au travail de la pulsion de mort, ce qui est en cause dans l'écriture de Claude Simon : comme l'indique Lucien Dällenbach à propos de La Route des Flandres, il s'agit de mettre en acte un " pouvoir d'envoûtement [...] d'empreinte, ainsi que nous le soufflent les mots eux-mêmes. ${ }^{6}$ ".

De même que $A u$ Cæuur des ténèbres se construit en anamorphose autour de Kurtz, le récit simonien ressasse une impossible scène : l'anéantissement de l'escadron de cavalerie dans Le Jardin des Plantes, la mort de Reixach dans La Route des Flandres, celle du père dans Le Tramway qui rend pour le fils toute conquête maudite. C'est un cataclysme où s'engouffre le désir humain, une nouvelle version de la scène primitive : non pas celle du coït parental (version freudienne) mais celle de la rencontre traumatique du réel de la pulsion tout entière livrée à son objet, quand les barrières de l'ordre symbolique ont sauté (version lacanienne). N'est-ce pas au choc d'un moment cristallisé dans le désastre de mai 1940 que Claude Simon voue son écriture : "J'étais hanté par deux choses : la discontinuité, l'aspect fragmentaire des émotions que l'on éprouve et qui ne sont jamais reliées les unes aux autres, et en même temps leur contiguïté dans la conscience $»^{7}$. Car il faut le dire : nous sommes au temps de la faillite des pères politiques, devenus machines de guerre (Rommel) ou de conquête économique (Kurtz) au profit d'une jouissance débridée, au plus près du fantasme du $(H) U n$ : figures hurlantes que l'on devine au plus près des semblants en décomposition, comme le pape de Francis Bacon. Le Jardin des Plantes évoque le masque de mort du Colonel dans l'ombre du caveau où pourrit le bel uniforme des pères orgueilleux de la nation, fiction de l'idéal enfoncé par la poussée métallique de la guerre. Pour l'artiste, la question sera de contenir (à la fois retenir et faire accueil) ce quelque chose de vaguement obscène par des « écrans qui constituent le sens en secret ${ }^{8}$ ", et ceci à double titre : le sens devient un secret qui travaille secrètement l'écrit.

\footnotetext{
${ }^{6}$ Ibid., p. 299.

7 Cité par L. Dällenbach, ibid., p. 301.

8 Ibid., p. 306.
} 
Sur ces écrans, une tache dévore de l'intérieur les lumières de l'Occident. De même que le visage de Marlow un instant animé par le rougeoiement d'un cigare se fond dans la nuit noire pour n'être plus qu'une onde vocale, celui des marins s'éclaire « d'en dessous par les fanaux posés à terre, inversant les ombres "; quelquefois les hommes-troncs cèdent l'Allée des Marronniers aux manèges et aux attractions des montagnes russes dont les lumières clignotent, " échafaudages compliqués d'où le grondement de catastrophe des wagonnets et les cris aigus des femmes venaient frapper la haute façade du monument aux morts" (Tram., 34) ; les humains ne sont que petits points agglutinés en surimpression, noircissant la plage devant le Casino alors que la mer scintille de gerbes d'eau " comme au passage de certains bancs de poissons frôlant la surface» (42). Mais on ne saura pas si les poissons sont passés : tout est surface, impression, scintillement argenté et mouvement décomposé comme sur les premières plaques photographiques. La substance du récit simonien, étrangement altérée, n'est d'ailleurs pas sans évoquer ces résumés de films affichant leurs "personnages en noir et blanc saisis dans des postures figées et privées de la magie du mouvement sur le scintillement argenté de l'écran " (33). Si bien qu'on s'interroge sur la filiation littéraire de cet étrange objet romanesque : "Roman de la mémoire, roman d'initiation, épopée, évocation lyrique ?9 ". Est-ce un monologue, un récit oral, écrit ? Les mêmes questions ont été maintes fois posées à propos de Conrad.

En tous cas, l'épopée a mal tourné : l'armée étrange et mythique des hommes-troncs, " exactes copies du même personnage [...] même visage osseux et dur de rapace " (Tram., 20) avec leurs corps martyrisés, s'affiche comme "une permanente récrimination à l'égard des vivants " (21) dont le monde glisse vers la zone de transit habitée par des "paires d'yeux sans expression dans les visages sans expression non plus, vides, comme absents " (27-28). L'érosion généralisée qui s'attaque aux façades de la bourgeoisie constitue une écriture, un symptôme de ce qui ronge la culture occidentale : de " minuscules scintillements de quartz " s'allument sous la lampe

9 Ibid., p. 303. 
torche dans la matière "friable, grenue et gris clair du crépi trop saturé de sable marin » de la maison de vacances (48-49). Et le tramway nommé désir, comme le steamer de Marlow avec ses hurlements déchirants dans le brouillard, s'anime parfois d'une méchante jouissance quand il laisse le narrateur en rade comme s'il "s'escamotait avec une sorte de ricanement moqueur et méchant " (33). Figure désabusée du guide, le wattman dont les gestes sont empreints de "quelque chose d'à la fois rituel et sacré " (14) forme avec le receveur une sorte de pseudo-couple flaubertien qui serait fait d'un même " personnage dédoublé » (18).

Comme dans Au cour des ténèbres, la femme est moins objet de désir que sœur d'un savoir sur la mort, et donc sœur d'armes des hommes-troncs. La mère du narrateur s'entête à porter son voile de crêpe " bien au-delà des limites décentes d'un deuil " (Tram., 21) ; son visage momifié fait penser " en féminisé, terreux et impitoyable, à ceux de ces amputés physiquement d'une moitié d'eux-mêmes " (23). Celle qui sait qu'elle va laisser un orphelin de onze ans, « macabre caricature de la voracité " avec son nez amaigri et osseux (38), ne fait pas couple avec l'homme mais avec la bonne au visage indéchiffrable (81) ou avec la tante qui affiche ce " même et long visage d'Erinye " (94) : elles sont liées par une connaissance commune du malheur comme les deux tricoteuses qui accueillent Marlow à l'entrée des bureaux de la compagnie coloniale : souvent, dira-t-il,

[...] je songeai à ces deux gardiennes de la porte des ténèbres, tricotant la laine noire, comme pour envelopper chaudement un cercueil, l'une faisant entrer, faisant entrer sans trêve dans l'inconnu, l'autre scrutant les visages réjouis et niais avec le détachement de ses yeux sans âge. Ave ! Vieille tricoteuse de laine noire. Morituri te salutant. $(A C T, 56)$

Plus loin dans son périple congolais Marlow contemple dans la case du comptable l'étude à l'huile d'une femme aux yeux bandés et à l'expression sinistre qui porte la torche des lumières de la civilisation ${ }^{10}$. La

\footnotetext{
10 " Je remarquai alors une petite étude à l'huile, sur un panneau, représentant une femme, drapée, les yeux bandés et portant une torche allumée. Le fond était ténébreux - presque noir. Le mouvement de la femme était majestueux, et la lueur de la torche sur le visage, sinistre. " $(A C T, 76)$.
} 
peinture "plus que défraîchie » intitulée «Les Mystères de la Femme » exhibe une figure féminine, sans doute l'une des grandes hystériques exposées au regard d'hommes de science,

[...] supposée attractive à la fois par la gravité « scientifique » de la scène représentée et, peut-on penser, par le fait qu'elle offrait aux regards le spectacle d'une femme demi-nue dont la chemise rejetée dévoilait une chair grisâtre [...]. (Tram., 54)

Mais la jouissance en cause ici relève moins de la complaisance à l'égard du fantasme masculin que d'un affichage de l'indicible, comme sur les panneaux des cinémas proposant aux spectateurs " les gigantesques visages de femmes échevelées, aux têtes renversées et aux bouches ouvertes dans un cri d'épouvante ou l'appel d'un baiser "(Tram., 14). Chez Simon comme chez Conrad il s'agit de déplacer ce désir de "ça-voir » vers quelque chose de beaucoup plus dérangeant, de faire vaciller l'écran de la représentation sur le secret vide qui nous regarde : car on n'entre pas dans le cinéma.

Le texte d'angoisse fait surgir non pas les monstres dévorateurs mais le silence d'un regard vide : ce désastre qui est la cause véritable du désir d'écrire. La Chose freudienne surgit en anamorphose, non pas comme chimère, Érinye, Méduse, mais comme point aveugle qui vous regarde dans les visages des malades « toujours inexpressifs, identiques, dans les masques figés, comme absents, des malades en attente [...] arrachés ou plutôt extirpés du monde familier, rassurant et multiple " (Tram., 30). Une pantomime très ancienne se joue sur la plage au devant du temple moderne, le Casino du Tramway: le spectacle vu de loin apparaît comme enrobé d'une totale absence de bruit (42-43). Plus loin la rêverie du narrateur étendu sur le pont d'une grosse barque, comme Marlow sur son steamer, laisse pénétrer le "silence noir " d'où surgit un fragment de corps entrevu à la lueur d'un fanal :

[...] pied que dans la demi-obscurité je distinguais à peine mais d'une telle présence quoique furtive (se posant tout près de mon visage pour disparaître aussitôt englouti par l'ombre) qu'il semblait appartenir à quelque divinité marine, non pas tant musculeux que noueux rongé par le sel [...]. (Tram., 73) 
On songe au bosquet obscur traversé par le silence des espaces infinis, où Marlow se retrouve le nez contre le coude anguleux d'un des travailleurs affamés de la cause inhumaine du progrès :

Les rapides étaient proches, et le fracas ininterrompu et uniforme des eaux précipitées remplissait la lugubre immobilité du bosquet, où l'on n'entendait pas un souffle, pas le frémissement d'une feuille, d'un son mystérieux - comme si la vitesse folle de la terre sur son orbite était soudain devenue audible. $(A C T, 64)$

De même chez Simon, la terre vue d'avion révèle l'errance cosmique de la matière "livrée à ses seules lois ", celle des plaques tectoniques, fragments d'écorce "s'attirant, se repoussant ou se fracassant dans une sauvage et majestueuse lenteur» (Tram., 125). Celle d'un monde non pas sans loi, mais où le réel fait loi.

C'est à ce traitement du réel que l'œuvre me semble-t-il se voue, comme pour en affronter la force intraitable mais sur un mode différent de celui de la science ou d'une littérature du souvenir. L'anamnèse simonienne est tout autre chose que la remémoration proustienne : elle ne cherche pas à faire surgir un univers entier d'une madeleine afin de retrouver une plénitude perdue. Au contraire, il s'agit de maintenir à distance sous le voile semi-transparent de la mémoire le lieu de la Chose où gît l'horreur. Mais de quoi le mot fait-il alors signe ? Il est moins pris dans un système de signification close que, par les vertus du son et du rythme, "branché " sur le souffle silencieux de la pulsion. Lécrivain travaille au littoral du signifiant bordé par ce que Lacan a appelé la langue du son, mémoire oublieuse faite d'échos commémorant un événement où le sujet retrouve une voix inconnue et pourtant familière. L'équivoque ou la simple résonance réveille un savoir venu du lieu de l'Autre du langage en chacun de nous, " en explorant les pouvoirs signifiants du mot "rideau" par exemple, au lieu de s'épuiser à voir ce qu'il y a derrière le voile ${ }^{11}$ ». Car ce qu'il y a, c'est précisément rien — res, rem, la Chose extime logée au cour du premier traumatisme du langage qui à son tour peut faire barrière et écho au rien. Du fait de sa double dimension de symbolique et de réel, un tel tissage sonore res-

${ }^{11}$ Lucien Dällenbach, op. cit. 
semble davantage à un habit d'Arlequin qu'à une tapisserie bien agencée. Il y faut des trous et des marques de suture que dessinent ces incessantes paralepses et métalepses d'un récit qui ne croit pas à son histoire : les incessants "as if ", " as though " de Marlow, les insistants " comme si » du narrateur du Tramway énoncent la sempiternelle inadéquation du langage au réel. Car il s'agit tout autant d'esquisser que d'esquiver ce réel.

Le voile est toujours linceul chez Conrad, il n'a pas vocation à être soulevé mais simplement à laisser s'écrire une part d'indicible sur la gaze de brume à la fois lumineuse et sinistre (" luminous" and " ominous") de l'incipit de Au cour des ténèbres. À cet égard la fin du Tramway est admirablement conradienne,

Comme si quelque chose de plus que l'été n'en finissait pas d'agoniser dans l'étouffante immobilité de l'air où semblait toujours flotter ce voile en suspension qu'aucun souffle d'air ne chassait, s'affalant lentement, recouvrant d'un uniforme linceul les lauriers touffus, les gazons brûlés par le soleil, les iris fanés et le bassin d'eau croupie sous une impalpable couche de cendres, l'impalpable et protecteur brouillard de la mémoire. (Tram., 141)

Brouillard prégnant d'un " quelque chose de plus ", d'un râle de jouissance et d'agonie venu du cœur des ténèbres, reconnu et tenu en respect par le voile de la mémoire. 